\title{
Novel vanadium phosphate phases as catalysts for selective oxidation
}

\author{
ARUNABHA DATTA*, MONIKA AGARWAL and \\ SOUMEN DASGUPTA \\ Indian Institute of Petroleum, Dehra Dun 248 005, India \\ e-mail: adatta@iip.res.in
}

\begin{abstract}
In our effort to induce novel modifications in the structure of some important vanadium phosphate phases used as selective oxidation catalysts, it has been observed that metal ions such as $\mathrm{Zn}^{2+}, \mathrm{Ni}^{2+}, \mathrm{Pd}^{2+}$ can be incorporated into the vanadyl hydrogen phosphate $\mathrm{VOHPO}_{4} \cdot 0 \cdot 5 \mathrm{H}_{2} \mathrm{O}$ phase in very different ways depending upon the medium of preparation. It has been found that the metal ions are either substituted into the lattice with retention of structure of the parent compound or intercalated between the layers of a new mixed-valent phase. These new metalincorporated phases are catalytically active and the palladium incorporated compound in particular displays shape selective catalysis for different oxidation and reduction reactions. In another approach, the preparation of $\mathrm{VOHPO}_{4} \cdot 0 \cdot 5 \mathrm{H}_{2} \mathrm{O}$ has been modified to give a novel crystalline phase containing mixed-valent vanadium and having $\mathrm{NH}_{3}$ species bound to the lattice. This phase could be a potential catalyst for ammoxidation reactions. In addition, novel mesostructured vanadium phosphate phases have been prepared using a long-chain amine as the templating agent involving a ligand templating mechanism of formation.
\end{abstract}

Keywords. Vanadium phosphate; selective oxidation; structure modification.

\section{Introduction}

One of the most important applications of selective oxidation catalysts is the functionalization of alkenes and aromatics because of the ease and economy with which they can be obtained from petroleum ${ }^{1}$. However, the trend now is to functionalize alkanes directly since they are relatively even more economical and readily available raw materials.

The major challenge in the selective oxidation of hydrocarbons is that thermodynamics favours total oxidation and the $\mathrm{C}-\mathrm{H}$ bonds in the initial reactant are generally stronger than those in the intermediate products which makes them prone to rapid further oxidation $^{2}$. The key step therefore is to stop the oxidation at the desired compound through kinetic control. This can be done only by designing the nature of active sites of the catalyst such that it is able to control the relative rates of different reaction pathways accelerating the steps leading to the desired product and hindering those which give unwanted products.

Presently, the only selective gas-phase alkane oxidation that has been commercialized is the oxidation of butane to maleic anhydride ${ }^{3}$. One major advantage in this reaction is that the stability of the product is higher than that of the starting alkane. However, the apparent paradox of catalytic chemistry, that higher yield and selectivity to maleic

*For correspondence 
anhydride are obtained in the oxidation of $n$-butane than in the oxidation of butenes, opens up the possibility that with a suitable choice of catalyst it may be possible to obtain a better performance in the selective oxidation of other alkanes rather than the corresponding alkene.

Catalysts used for selective oxidation are generally oxides, and vanadium and molybdenum oxides are the most widely used ones for the selective oxidation of alkanes. Amongst these the most preferred element is vanadium. A vanadium pyrophosphate phase $(\mathrm{VO})_{2} \mathrm{P}_{2} \mathrm{O}_{7}$ is commercially used as catalyst for the selective oxidation of butane to maleic anhydride. Vanadium phosphorous oxides (VPO) have also shown promising results as heterogeneous catalysts in the selective oxidation of propane to acrylic acid, pentane to maleic and pthalic anhydride, and in the oxidative dehydrogenation of ethane, propane etc ${ }^{4}$.

Vanadium phosphates constitute a very interesting class of layered compounds which exist in a wide range of structural forms both due to the variable valency of vanadium as well as the large diversity in the bonding of the $\mathrm{VO}_{6}$ octahedron and the $\mathrm{PO}_{4}$ structural units ${ }^{5}$. Several phases containing vanadium in $+5,+4$ and +3 oxidation states are known. In all these compounds the layers are held together by hydrogen bonding or by weak Van der Waal's interactions. These layered compounds are therefore amenable for intercalation reactions.

In the present work we describe different approaches that have been adopted to synthesise novel phases in the VPO system as well as modify the structures of some known catalytically important vanadium phosphate phases. In addition the catalytic activity of some of these new and modified phases have been investigated.

\section{Experimental section}

\subsection{Preparation of metal incorporated phases}

The $\mathrm{VOHPO}_{4} \cdot 0 \cdot 5 \mathrm{H}_{2} \mathrm{O}$ phase was prepared by a published method ${ }^{6 \mathrm{a}}$ involving the reduction of an aqueous slurry of $\mathrm{V}_{2} \mathrm{O}_{5}$ with $\mathrm{NH}_{2} \mathrm{OH} \cdot \mathrm{HCl}$ followed by the addition of a stoichiometric amount of $85 \%$ phosphoric acid $(\mathrm{P}: \mathrm{V}=1: 1)$. In the preparation of the intercalation compounds, the relevant metal salt was added to the $\mathrm{V}_{2} \mathrm{O}_{5}$ slurry (V/M = 1:0.2, M = Zn, Ni, Pd). The zinc, nickel and palladium incorporated compounds are coded as $\mathbf{A} / \mathbf{Z n}, \mathbf{A} / \mathbf{N i}$ and $\mathbf{A} / \mathbf{P d}$ respectively. However, in the synthesis in organic medium ${ }^{6 \mathrm{~b}}$, the reduction of $\mathrm{V}_{2} \mathrm{O}_{5}$ was carried out by isobutanol-benzyl alcohol mixture and the relevant metal salts were added in the same atomic ratio of $\mathrm{P}: \mathrm{V}: \mathrm{M}=1: 1: 0 \cdot 2$. The metal intercalates were coded as $\mathbf{O} / \mathbf{Z n}, \mathbf{O} / \mathbf{N i}$ and $\mathbf{O} / \mathbf{P d}$.

\subsection{Synthesis of new phases through modification of conventional procedures}

For the preparation of a new phase the conventional method for the preparation of $\mathrm{VOHPO}_{4} \cdot 0 \cdot 5 \mathrm{H}_{2} \mathrm{O}$ in aqueous medium was modified as described above by introducing a time delay between the onset of the reduction of $\mathrm{V}_{2} \mathrm{O}_{5}$ with hydroxylamine hydrochloride and the addition of phosphoric acid. The final phases were then isolated in the usual manner as described above in the preparation of $\mathrm{VOHPO}_{4} \cdot 0 \cdot 5 \mathrm{H}_{2} \mathrm{O}$. The phases obtained at different delay times of 0 to $10 \mathrm{~min}$ are referred to as $\mathbf{P - 0}, \mathbf{P}-\mathbf{1}, \mathbf{P}-\mathbf{2}, \mathbf{P}-\mathbf{3}, \mathbf{P}-\mathbf{4}, \mathbf{P}-\mathbf{5}, \mathbf{P}-6$, P-7, P-8, P-9, P-10 respectively. 


\subsection{Preparation of mesostructured phases}

Mesostructured vanadium phosphates using hexadecylamine (HDA) as the templating agent were synthesized from a reaction mixture of the following molar composition, $\mathrm{V}_{2} \mathrm{O}_{5}: \mathrm{H}_{3} \mathrm{PO}_{4}: \mathrm{NH}_{2} \mathrm{OH} \cdot \mathrm{HCl}: \mathrm{HDA}: \mathrm{H}_{2} \mathrm{O}=1: 2: 2: 0 \cdot 5: 669$. In a typical synthesis, $\mathrm{V}_{2} \mathrm{O}_{5}$ $\left(0.909 \mathrm{~g}, 0.005\right.$ mole) powder was stirred in $30 \mathrm{ml}$ aqueous solution of $\mathrm{NH}_{2} \mathrm{OH} \cdot \mathrm{HCl}$ $(0.695 \mathrm{~g}, 0.01 \mathrm{~mole})$ in presence of $1.153 \mathrm{~g}$ of $85 \%$ phosphoric acid $(0.01 \mathrm{~mole})$ at $80^{\circ} \mathrm{C}$ for $1 \mathrm{~h}$ to get a blue solution. Into it a homogeneous dispersion of HDA ( $0.603 \mathrm{~g}, 0.0025$ mole) in $30 \mathrm{ml}$ water was added. Immediate precipitation of a sky blue solid was observed. The solid was separated from the reaction mixture after stirring for $1 \mathrm{~h}$ at $80^{\circ} \mathrm{C}$ and was thoroughly washed with boiling water followed by acetone to remove any unreacted HDA. This material was designated as A-1. In another experiment, the precipitated solid was separated from the mother liquor after $24 \mathrm{~h}$ of stirring at $80^{\circ} \mathrm{C}$ while keeping all other reaction conditions the same as in the case of A-1. This material was named A-2.

\section{Results and discussions}

\subsection{Metal incorporated vanadium phosphates}

It has been observed ${ }^{7}$ that when metal ions such as $\mathrm{Zn}^{2+}, \mathrm{Ni}^{2+}, \mathrm{Pd}^{2+}$ are incorporated into the $\mathrm{VOHPO}_{4} \cdot 0 \cdot 5 \mathrm{H}_{2} \mathrm{O}$ phase during its preparation in organic medium, the basic structure of the parent compound is retained, as evident from their XRD patterns (figure 1). In the

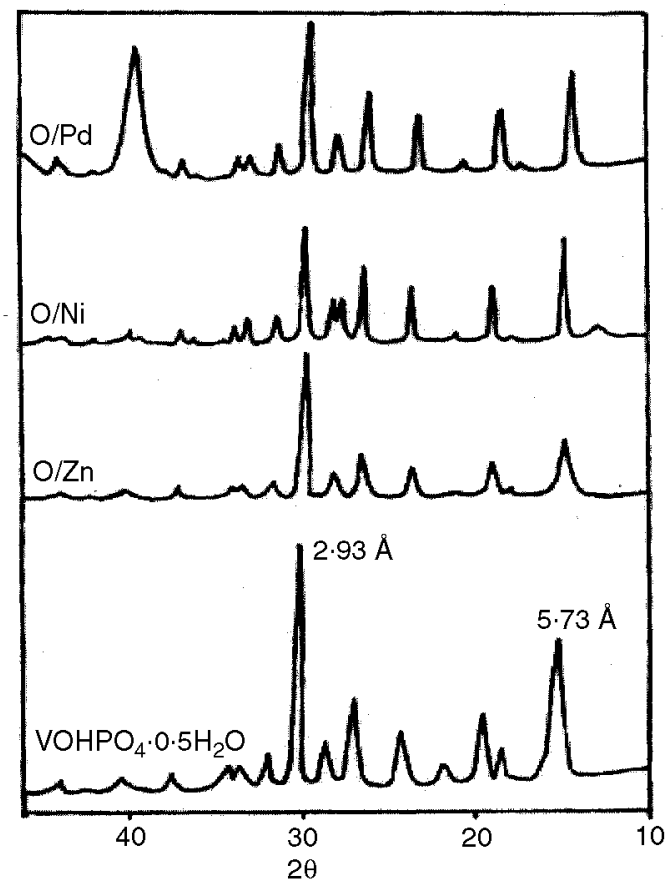

Figure 1. XRD patterns of compounds $\mathrm{VOHPO}_{4} \cdot 0 \cdot 5 \mathrm{H}_{2} \mathrm{O}, \mathbf{O} / \mathbf{Z n}, \mathbf{O} / \mathbf{N i}, \mathbf{O} / \mathbf{P d}$ prepared in organic medium ( $\mathrm{CuK} \alpha$ radiation). 
case of O/Pd though there is an additional peak due to the $100 \%$ line of metallic palladium. All these phases also undergo transformation to the pyrophosphate phase although the transformation temperatures are slightly higher in the metal incorporated phases $\left(452^{\circ} \mathrm{C}, 465^{\circ} \mathrm{C}\right.$ and $454^{\circ} \mathrm{C}$ for $\mathbf{O} / \mathbf{Z n}, \mathbf{O} / \mathbf{N i}$ and $\mathbf{O} / \mathbf{P d}$ respectively, as compared to $443^{\circ} \mathrm{C}$ for the parent compound). The infrared spectra of these samples are also essentially similar to that of the parent compound.

On the other hand, when the same metal ions are introduced during the preparation of the hemihydrate by the reduction of $\mathrm{V}_{2} \mathrm{O}_{5}$ with hydroxylamine hydrochloride in aqueous medium, altogether new phases are formed ${ }^{8}$ whose XRD patterns (figure 2) show very

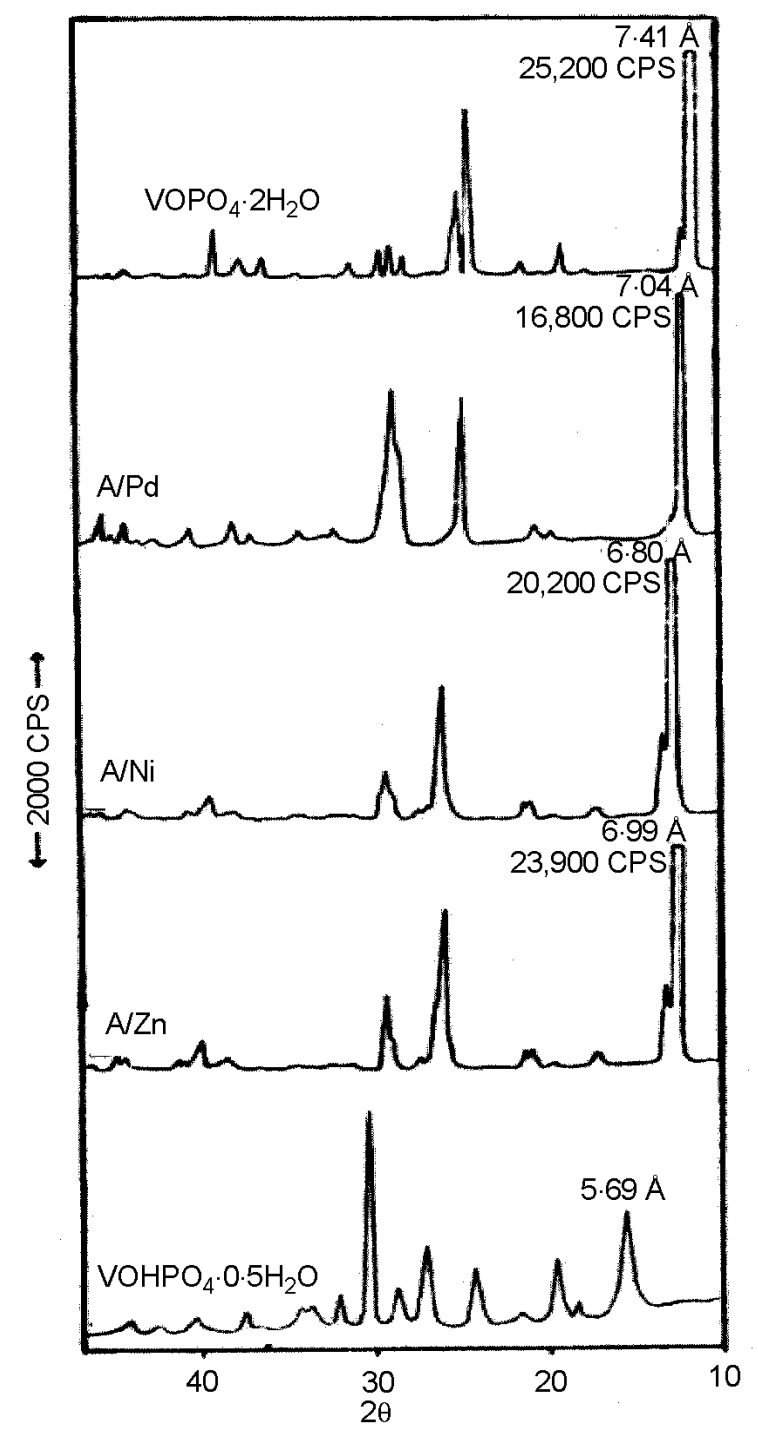

Figure 2. XRD patterns of compounds $\mathrm{VOHPO}_{4} \cdot 0 \cdot 5 \mathrm{H}_{2} \mathrm{O}, \mathbf{A} / \mathbf{Z n}, \mathbf{A} / \mathbf{N i}, \mathbf{A} / \mathbf{P d}$ prepared in aqueous medium $(\mathrm{CuK} \alpha$ radiation). 
selective ordering along the $c$-axis. All the three intercalates have a similar pattern with the interlayer distance varying with the metal ion from $6.80 \AA$ in the nickel compound to $7.04 \AA$ in the case of the palladium intercalate. These phases do not transform to the pyrophosphate phase and have FTIR spectra very different from that of the parent compound. The average vanadium oxidation state of these samples is +4.65 indicating mixed valency. This is further confirmed by their X-ray photoelectron spectra (XPS) which show a broad peak which can be deconvoluted into two peaks corresponding to $\mathrm{V}^{4+}$ and $\mathrm{V}^{5+}$ species.

It is shown therefore that the metal ions $\mathrm{Zn}^{2+}, \mathrm{Ni}^{2+}$ and $\mathrm{Pd}^{2+}$ can be incorporated into the $\mathrm{VOHPO}_{4} \cdot 0 \cdot 5 \mathrm{H}_{2} \mathrm{O}$ phase in very different ways depending upon the medium of preparation. In the synthesis in organic medium the structure of the parent compound is retained and the metals appear to substitute into the lattice. On the other hand in aqueous medium the same metal ions induce the formation of a new mixed valent vanadium phosphate phase with the metal ions lodged in the interlayer region.

Since the metal ions are incorporated in very different ways into the vanadium phosphate phase, the catalytic activity of these incorporated metal ions was investigated. It was found for instance that in the nickel catalyzed dehydrogenation of amines to nitriles, on stirring an aqueous solution of the catalyst with benzylamine (Cat: Substrate = 1:200) in $\mathrm{CH}_{2} \mathrm{Cl}_{2}$ and an aqueous solution of $\mathrm{K}_{2} \mathrm{~S}_{2} \mathrm{O}_{8} / \mathrm{NaOH}$ for $6 \mathrm{~h}$ at room temperature, $92 \%$ benzonitrile was obtained in the case of $\mathbf{O} / \mathbf{N i}$ whereas no conversion to the benzonitrile was observed ${ }^{9}$ in the case of $\mathbf{A} / \mathbf{N i}$.

The palladium compounds also showed very dramatic differences in their catalytic activity ${ }^{10}$. In the oxidative coupling of furan and ethyl acrylate $\mathbf{O} / \mathbf{P d}$ and $\mathbf{A} / \mathbf{P d}$ gave $32 \%$ and $13 \%$ of the monosubstituted product only compared to $11 \%$ and $5 \%$ of the mono- and di-substituted products respectively in the case of palladium acetate, the conventionally used homogenous catalyst for this reaction. This clearly indicated that both $\mathbf{O} / \mathbf{P d}$ and A/Pd display shape selectivity by catalysing the formation of the mono-substituted derivative only. Also, O/Pd in the heterogenous mode gives selectively three times higher yield of the mono-substituted product as compared to the conventionally used homogenous catalyst. On the other hand in the hydrogenation of nitrobenzene to aniline, A/Pd gave the product in nearly quantitative yield but $\mathbf{O} / \mathbf{P d}$ did not yield any aniline at all. Also, in the hydrogenation of cinnamaldehyde, A/Pd gave $72 \%$ and $28 \%$ of the saturated aldehyde and alcohol respectively whereas, with $\mathbf{O} / \mathbf{P d}$ as the catalyst, an almost equimolar mixture of the two products was obtained.

\subsection{Novel phases through modification of conventional preparation procedures}

In addition to modifying the structure of the vanadyl hydrogen phosphate phase through metal incorporation, we have also obtained ${ }^{11}$ a new mixed valent phase by a slight modification of the preparation of the hemihydrate. In the normal preparation of $\mathrm{VOHPO}_{4} \cdot 0 \cdot 5 \mathrm{H}_{2} \mathrm{O}$ through reduction of $\mathrm{V}_{2} \mathrm{O}_{5}$ using hydoxylamine hydrochloride, both the reductant and phosphoric acid are added at the same time. However, by introducing a time delay between the onset of reduction and the addition of phosphoric acid a whole series of different phases are obtained.

It can be seen from the XRD pattern (figure 3) of phases prepared with delay times in the range 0 to $10 \mathrm{~min}(\mathbf{P - 0}$ to $\mathbf{P - 1 0})$ that initially up to delay times of $3 \mathrm{~min}(\mathbf{P}-\mathbf{0}$ to $\mathbf{P - 3})$ the pure hemihydrate phase is obtained. However the phase P-4 is a disordered VOHPO $_{4} \cdot 0 \cdot 5 \mathrm{H}_{2} \mathrm{O}$ phase while phases P-5 and P-6 are X-ray amorphous. At 7 min delay 
time a new crystalline phase appears which becomes more ordered when delay times of 8, 9 and $10 \mathrm{~min}$ are employed. The XRD pattern of the latter phase (P-10) is completely different from that of $\mathrm{VOHPO}_{4} \cdot 0 \cdot 5 \mathrm{H}_{2} \mathrm{O}$ or any of the other known phases in the VPO system.

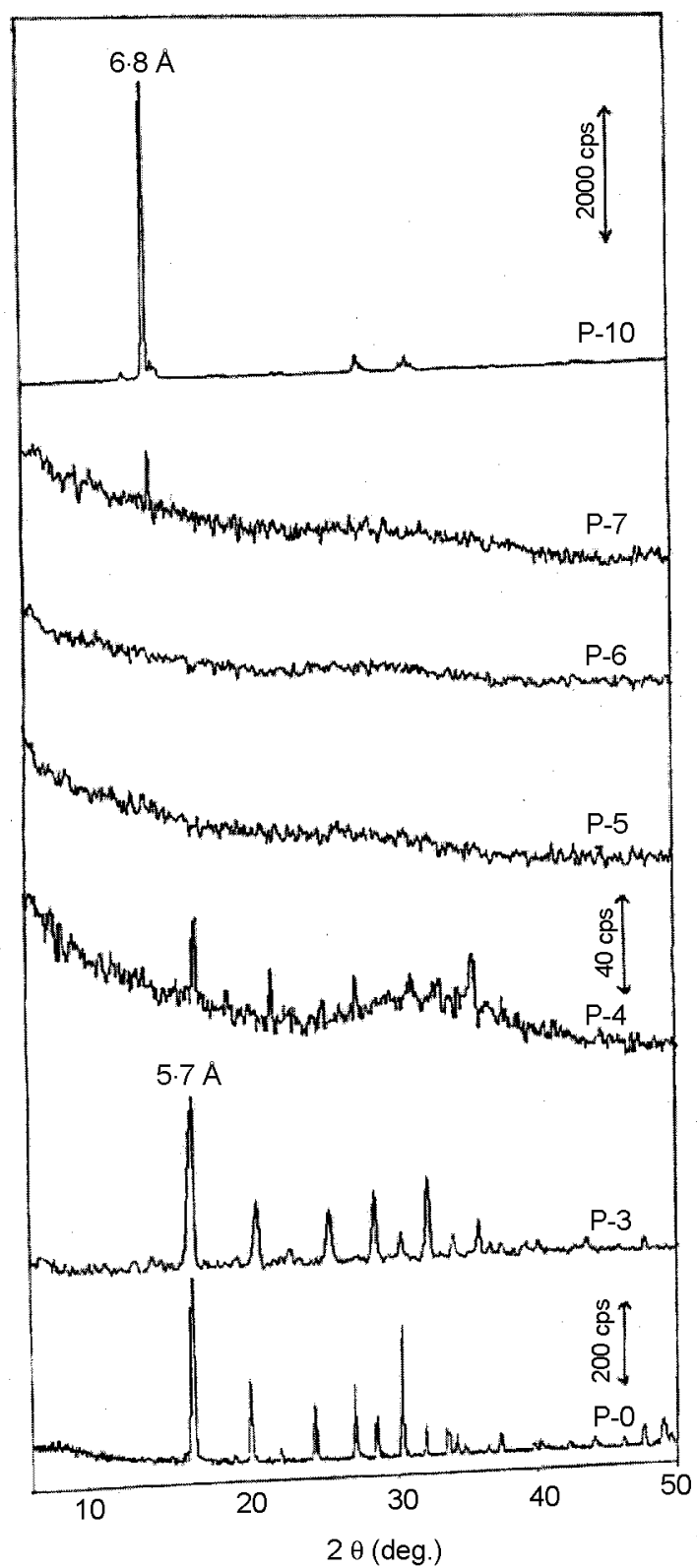

Figure 3. XRD patterns of phases prepared with different delay times $(\mathrm{CuK} \alpha$ radiation). 

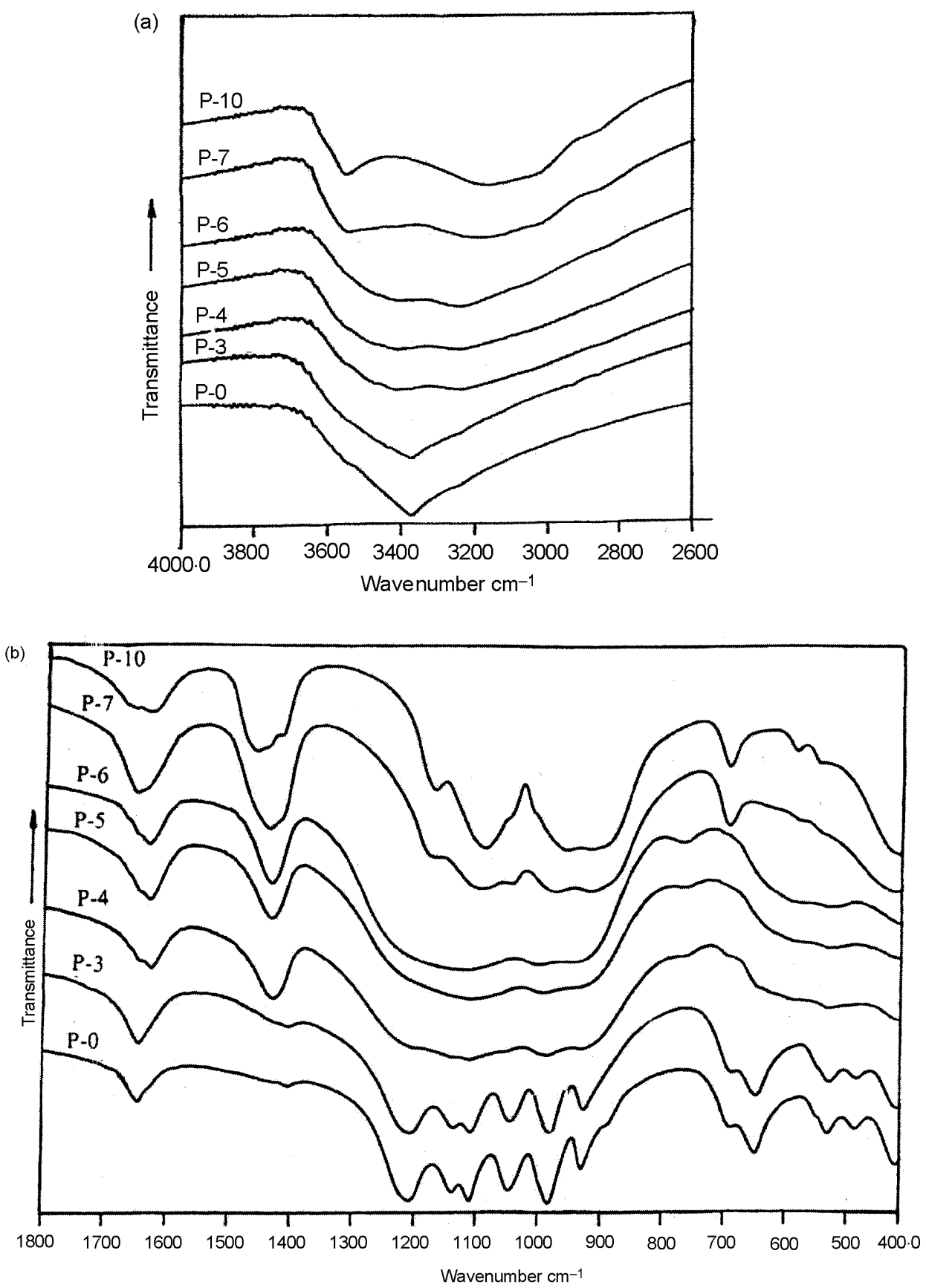

Figure 4. FTIR spectra of phases prepared with different delay times: (a) $\mathrm{O}-\mathrm{H}$ stretching region; (b) $\mathrm{P}-\mathrm{O}, \mathrm{V}-\mathrm{O}$ stretching region.

The FTIR spectra (figure 4) corroborate the findings the XRD data of the phases P-0 to $\mathbf{P}-3$ corresponding to that reported for $\mathrm{VOHPO}_{4} \cdot 0 \cdot 5 \mathrm{H}_{2} \mathrm{O}$ phase. Interestingly, the onset of 
disorder in the $\mathrm{VOHPO}_{4} \cdot 0 \cdot 5 \mathrm{H}_{2} \mathrm{O}$ and the subsequent formation of the $\mathrm{X}$-ray amorphous phases P-4 and P-5 are associated with the appearance of bands at 1610 and $1425 \mathrm{~cm}^{-1}$, which correspond to the $\mathrm{N}-\mathrm{H}$ bending modes of $\mathrm{NH}_{3}$ bound to Lewis and Bronsted acid sites respectively ${ }^{12}$. Similar bands are also present in P-7 and in P-10 and occur at $1615 \mathrm{~cm}^{-1}$, and at 1444 and $1410 \mathrm{~cm}^{-1}$. In essence it is apparent that the presence of bound $\mathrm{NH}_{3}$ causes the loss in crystallinity of the initially formed $\mathrm{VOHPO}_{4} \cdot 0 \cdot 5 \mathrm{H}_{2} \mathrm{O}$ and the subsequent formation of an amorphous phase and the eventual emergence of a new crystalline phase, $\mathbf{P - 1 0}$.

(a)

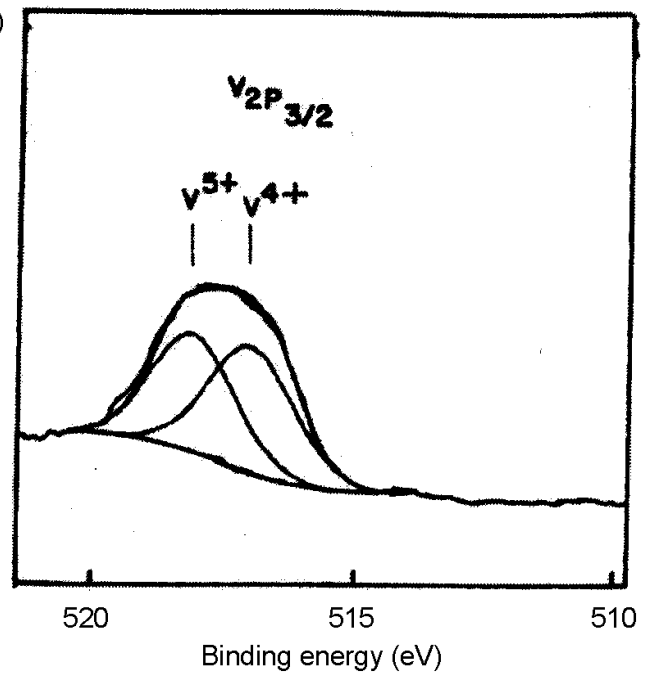

(b)

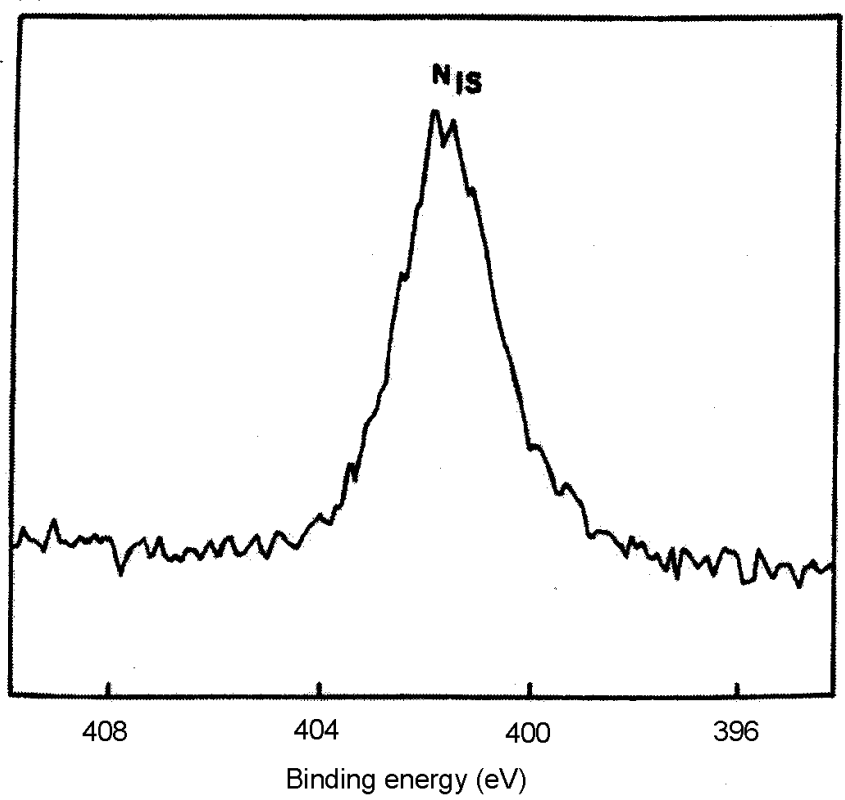

Figure 5. XPS of P-10, (a) $\mathrm{V}_{2 \mathrm{p} 3 / 2}$ and (b) $\mathrm{N}_{1 s}$ region. 
The characterization of P-10 shows that it contains mixed-valent vanadium as evident from its XPS spectrum (figure 5) which shows a broad peak resolved into two peaks with binding energies of 518.0 and $516.9 \mathrm{eV}$ due to $\mathrm{V}^{3+}$ and $\mathrm{V}^{4+}$ species respectively. In addition, the presence of $\mathrm{NH}_{4}^{+}$type species is indicated by an $\mathrm{N}_{1 s}$ peak at $401.6 \mathrm{eV}$. Mixed valency of vanadium is also indicated by a unique band at $740 \mathrm{~nm}$ in its UV-Vis reflectance spectrum which can be assigned to charge transfer between $\mathrm{V}^{4+}$ and $\mathrm{V}^{5+}$ species. Also the TGA pattern of P-10 indicates that it does not undergo transformation to the pyrophosphate phase but loses 1.8 molecules of water at temperatures below $200^{\circ} \mathrm{C}$ to give an amorphous phase. The EPR spectrum of P-10 shows a sharp signal with a peak width of $30 \mathrm{G}$ centred at $g_{1}=1.9708$ overlapping a broader signal with $g_{2}=1.9574$, thus indicating the presence of two types of $\mathrm{V}^{4+}$ ions with different environments.

The in situ generation of $\mathrm{NH}_{3}$ from the hydroxylamine hydrochloride is itself a novel and intriguing aspect of the chemistry of VPO compounds. It would appear that a delay in the addition of phosphoric acid leads to an accumulation of $\mathrm{V}^{4+}$ ions which activates the $\mathrm{N}^{1-} / \mathrm{N}^{3-}$ couple $\left(E^{\circ}=+1.35 \mathrm{~V}\right)$ of $\mathrm{NH}_{2} \mathrm{OH} \cdot \mathrm{HCl}$ and oxidises $\mathrm{V}^{4+}$ to $\mathrm{V}^{5+}\left(E^{\circ}=-0.957 \mathrm{~V}\right)$ and generates $\mathrm{NH}_{3}$ in the process. After delay times of 4 min the liberated ammonia causes the hemihydrate phase to become amorphous, most likely through interaction with

(b)

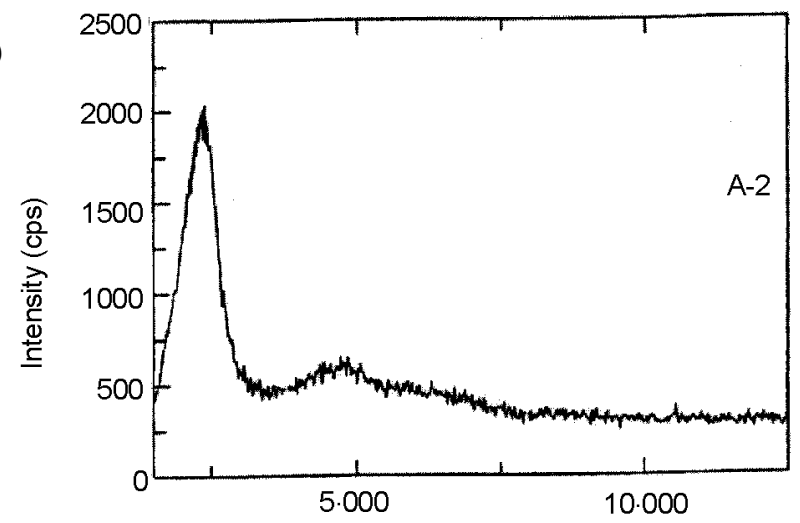

(a)

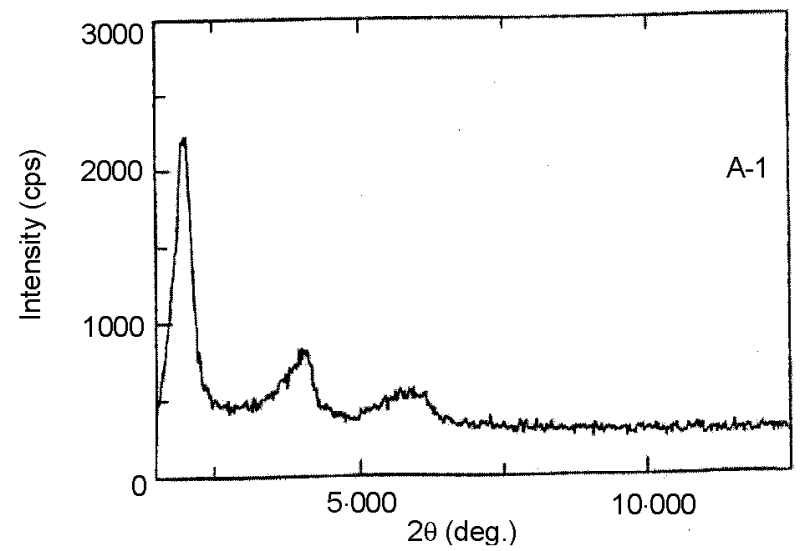

Figure 6. XRD patterns of HDA templated mesostructured vanadium phosphates A-1 (1 h) (a) and A-2 (24 h) (b) (CuK $\alpha$ radiation). 
P-OH groups (Bronsted acid sites) and the vanadium (Lewis acid sites). Subsequently the ammonia is incorporated into the new crystalline mixed valent phase. This is a very novel aspect of VPO chemistry since earlier studies indicate that interaction of ammonia with crystalline VPO phases invariably makes them amorphous ${ }^{13}$.

\subsection{Mesostructure induction in vanadium phosphate}

One of the major areas of research in the quest for new catalytic materials is the synthesis of new open structures with pores in the mesoporous range as well as lamellar phases of

(a)

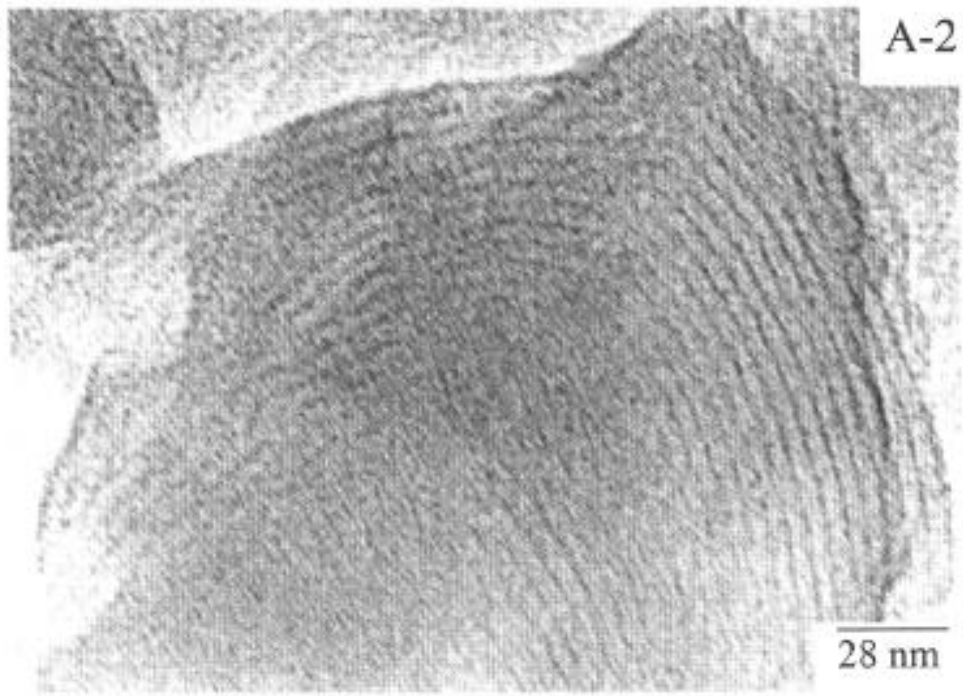

(b)

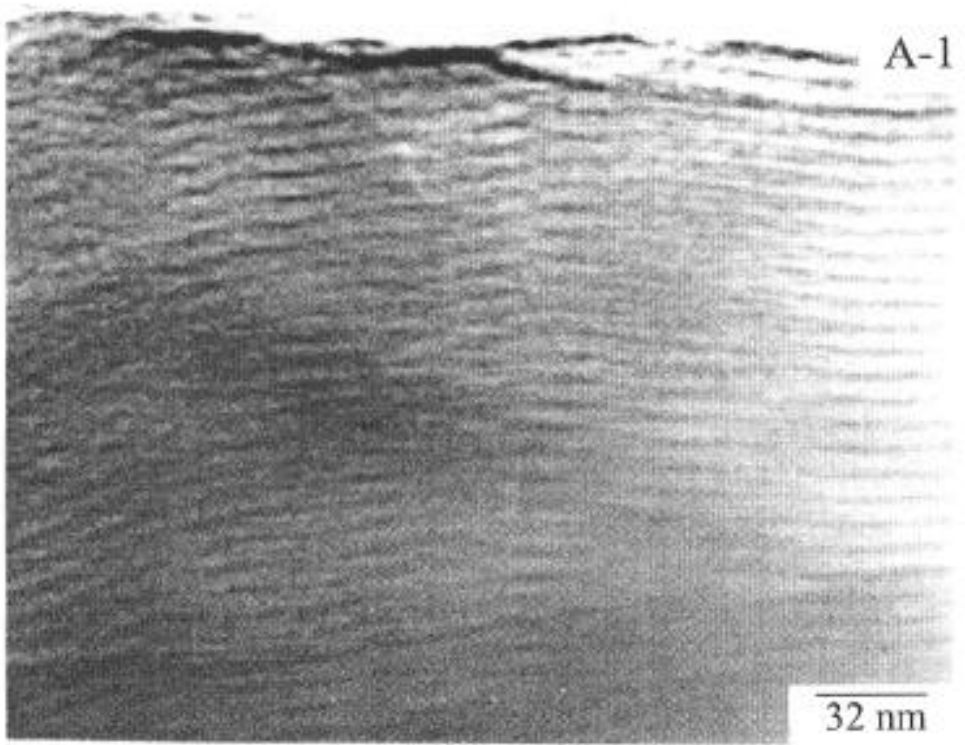

Figure 7. TEM of A-1 and A-2 (accelerating voltage, $200 \mathrm{kV}$ ). 
mesoscopic dimensions ${ }^{14}$. To this end the synthesis by researchers ${ }^{15}$ at Mobil in 1992 of mesostructured silicates (M41S) using long chain surfactants as supramolecular templates opened new horizons in this area and this templating method has now been applied to a number of non-silica based materials as well. Some attempts have recently also been made to induce mesostructure formation in vanadium phosphates ${ }^{16}$. In this context, we have synthesized a mesoscopic vanadium phosphate phase by the templating action of a long chain alkyl amine surfactant such as hexadecylamine (HDA) ${ }^{17}$.

The formation of mesostructured phases is evident from the XRD pattern (figure 6) of A-1 which shows a strong diffraction peak at $d=44.5 \AA$ and two other peaks of weaker intensity at $d=22.2$ and $14.8 \AA$ respectively. These can be assigned to the (001), (002) and (003) reflections respectively of a mesolamellar phase with a basal spacing of $44.5 \AA$. Interestingly, in the case of A-2, the basal spacing decreased to $38.04 \AA$ and only one other diffraction peak with a $d$ value of $18.9 \AA$ and assignable to the (002) reflection was observed. This indicated restructuring of the incorporated HDA molecules on aging.

The transmission electron micrograph (TEM) of A-1 (figure 7) shows parallel stacking of layers with interlayer spacing of $44 \cdot 1 \AA$ which corresponds well with the basal spacing of $44.5 \AA$ obtained from XRD data. In the case of A-2, a considerable degree of curvature in the layer structure is introduced with a concomitant decrease in the layer spacing to $38.08 \AA$ (38.04 A from XRD data).

It is evident therefore that it is possible to organize soluble vanadium phosphate precursors into mesolamellar $\mathrm{V}-\mathrm{P}-\mathrm{O}$ phases by using a long chain amine such as HDA as the template. FTIR studies suggest strong interaction of the incorporated HDA molecules in the free amine form with the VPO matrix, thus suggesting a ligand templating mechanism of mesostructure formation.

\section{Conclusions}

It has been demonstrated that the structures of vanadium phosphate phases which are catalytically important for the selective oxidation of hydrocarbons, can be modified by various different approaches.

(1) Metal ions can be incorporated into the catalytically important vanadyl hydrogen phosphate phase in different ways depending upon the medium of preparation. These constitute a novel class of compounds in which oxidation centres are confined within the layers of a parent oxidation catalyst. This gains significance in light of the belief that selective oxidation catalysts require different sites for activity and selectivity. The palladium incorporated compounds in particular show good catalytic activity and also display shape selectivity. In addition, the substitution of divalent metal ions into the lattice of the parent compound most likely by replacing (VO) ${ }^{2+}$ species (to maintain charge neutrality) would cause oxygen vacancies which are known to be active sites for lattice oxygen-mediated selective oxidation catalysis.

(2) By modifying the procedure for the preparation of a commercially important vanadium phosphate, it has been possible to obtain for the first time a crystalline vanadium phosphate phase with an incorporated $\mathrm{NH}_{3}$ species. The in situ generation of $\mathrm{NH}_{3}$, during the use of $\mathrm{NH}_{2} \mathrm{OH} \cdot \mathrm{HCl}$ as a reductant, is itself a very novel aspect of VPO chemistry and the present results demonstrate that it is possible to exploit the very rich solution chemistry of vanadium compounds for making novel phases. The $\mathrm{NH}_{3}$ incorporated phase could be a strong candidate for ammoxidation catalysis. On the other 
hand, the intermediate phases obtained with delay times of 4-7 min, which were found to give rise to disordered pyrophosphate phases, could function as good catalysts for selective oxidation of hydrocarbons since it has been shown that disordered phases exhibit higher catalytic activity.

(3) The preparation of mesostructured vanadium phosphates is encouraging since there are very few reports of such phases. Also it raises interesting possibilities such as the incorporation of large metal complexes which are active homogeneous catalysts, into mesostructured vanadium phosphate phases. This would lead not only to the heterogenization of homogenous systems but could also produce novel catalysts.

\section{References}

1. Albonetti S, Cavani F and Trifiro F 1996 Catal. Rev. Sci. Eng. 58413

2. Cassidy F E and Hodnett B K 1998 Catal. Tech. 2173

3. Centi G, Trifiro F, Ebner J R and Franchetti V M 1988 Chem. Rev. 8855

4. Centi G (ed.) 1993 Catal. Today 165, and references therein

5. Bordes E 1988 Catal. Today 3163

6. (a) Shimoda T, Okuhara T and Misono M 1985 Bull. Chem. Soc. Jpn. 58 2163; (b) Busca G, Cavani F, Centi G and Trifiro F 1986 J. Catal. 99400

7. Datta A, Kelkar R Y and Saple A R 1994 Proc. Indian Acad. Sci. (Chem. Sci.) 106629

8. Datta A, Kelkar R Y and Saple A R 1994 J. Chem. Soc., Dalton Trans. 2145

9. Datta A, Saple A R and Kelkar R Y 1991 J. Chem. Soc., Chem. Commun. 1645

10. Datta A, Agarwal M and Dasgupta S 2002 J. Mol. Catal. A181 119

11. Datta A, Agarwal M and Dasgupta S 2002 J. Mater. Chem. 121892

12. Zhang Y, Martin A, Berndt H, Lücke B and Meisel M 1997 J. Mol. Catal. A118 205

13. (a) Zhang Y, Martin A, Wolt G V, Rabe S, Worzala H, Lücke B, Meisel M and Witke K 1996 Chem. Mater. 8 1135; (b) Zhang Y, Meisel M, Martin A, Lücke B, Witke K and Brzezinka K W 1997 Chem. Mater. 91086

14. Sayari A and Liu P 1997 Microporous Mater. 12149

15. Beck J S, Vartuli J C, Roth W J, Leonowicz M E, Kresge C T, Schmitt K D, Chu C T-W, Olson D H, Sheppard E W, McCullen S B, Higgins J B and Schlenker J L 1992 J. Am. Chem. Soc. 11410834

16. (a) Abe T, Taguchi A and Iwamoto M 1995 Chem. Mater. 7 1429; (b) Roca M, Haskouri J E, Cabrera S, Beltrán-Porter A, Alamo J, Beltrán-Porter D, Marcos M D and Amorós P 1998 Chem. Commun. 1883; (c) Haskouri J E, Roca M, Cabrera S, Alamo J, Beltrán-Porter A, Beltrán-Porter D, Marcos M D and Amorós P 1999 Chem. Mater. 11 1446; (d) Mizuno N, Hatayama H, Uchida S and Taguchi A 2001 Chem. Mater. 13 179; (e) Doi T and Miyake T 1996 Chem. Commun. 1635; (f) Haskouri J E, Cabrera S, Roca M, Alamo J, Beltrán-Porter A, Beltrán-Porter D, Marcos M D and Amoros P 1999 Inorg. Chem. 384243

17. Dasgupta S, Agarwal M and Datta A 2002 J. Mater. Chem. 12162 